Communication présentée

au Comité Technique de la Société Hydrotechnique de France le 20 mars 1964

UN PHYSICIEN AUX PRISES AVEC LES SOURCIERS

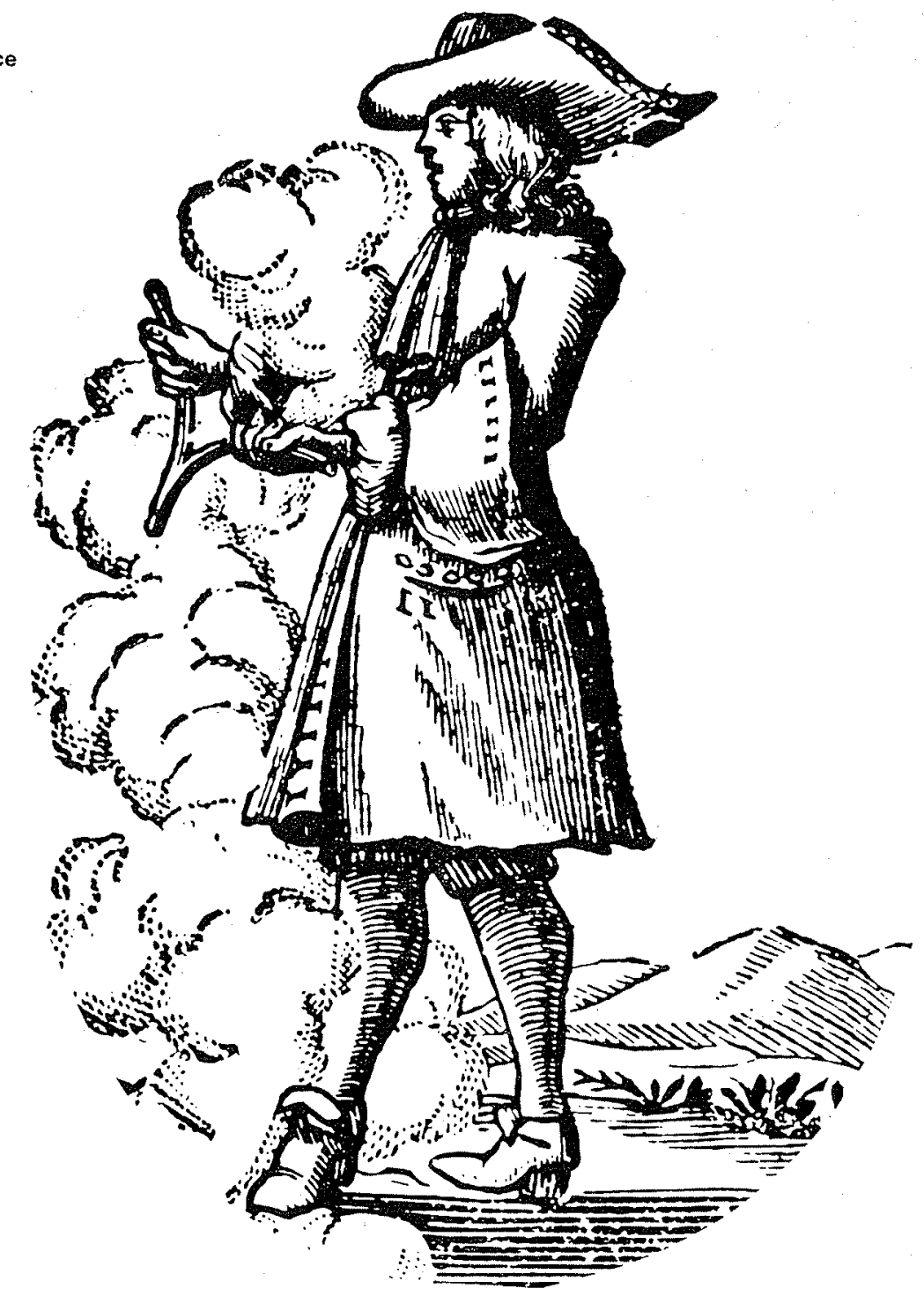

mes saugrenus et exige une prudence sur laquelle j'attire respectueusement votre attention sans plus tarder.

Chacun, dans sa famille, avait autrefois un sourcier valable, et j'espère bien que beaucoup d'entre vous ont une expérience tout à fait indépendante de ce que je vais raconter. Toutefois, la jeune génération vit surtout dans des automobiles, engins perchés sur des roues isolantes et qui restent sur du macadam : j'ai bien peur que l'expérience sourcière diminue dans cette génération... Autrement dit, on a moins, dans la vie moderne, l'occasion de trouver des signaux authentiques sur le terrain dans des conditions valables.

L'art du sourcier n'est pas aussi ancien qu'on veut bien le dire. On peut toujours faire des remarques sur les baguettes que les pharaons tiennent dans leurs tombeaux, du moins sur les peintures; la verge de Moïse est peut-être une baguette de sourcier.

En fait, c'est vers 1450 que l'on trouve les premiers documents où l'on voit un homme avec une baguette en position et il semble que l'affaire soit née chez les mineurs allemands. A l'Ecole des Mines de Heidelberg, en 1500, on trouve des thèses soutenues sur la baguette, dont l'emploi paraissait fort naturel alors et n'était l'objet d'aucune contestation.
* Professeur à la Sorbonme, Paris.

Le dessin qui illustre cet article est extrait de l'ouvrage de Y. Rocand: Le Signal du Sourcier (2" édition, 1964, Dunod éditeur). 
Ces mineurs allemands cherchaient plutôt les métaux. Ils ont été introduits en France par François Ier, qui en avait fait venir une cinquantaine avec un certain baron de Beausoleil, lequel s'est fait un nom en découvrant beaucoup de mines, cataloguées dans un ancien ouvrage de Nicolas Gobet: Les anciens métallurgistes, livre très valable, qui énumère 150 ou 200 mines trouvées en France un peu partout.

En Angleterre, même opération: les mineurs allemands sont venus dans ce pays à la même époque. C'est à peu près à ce moment que l'on a conclu que la baguette marchait mieux sur l'eau et que l'on a un peu abandonné les métaux.

Subitement, ver's 1680, un paysan du Dauphiné devenu célèbre par cette aflaire, Jacques Aymar, en recherchant des objets volés, descend dans une cave et découvre un cadavre: la baguette tournait sur ce cadavre.

Le voilà donc embauché par la police du Roi et prié, peut-être avec quelques menaces à l'appui, de trouver un coupable. Et il en a trouvé un en faisant une odyssée extraordinaire qui l'a fait descendre de son pays grenoblois, à travers toute la vallée du Rhòne. Dans une prison à Beaucaire, il trouve un pauvre diable qui avoue être l'auteur du crime : la baguette de Jean Aymar toumait quand il posait son pied sur celui d'un coupable...

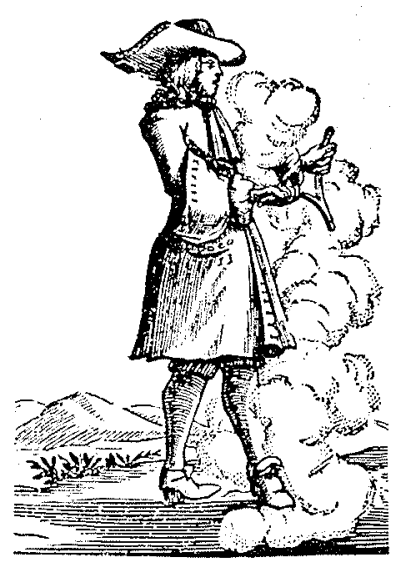

Cet événement a subitement créé un intérêt immense sur les possibilités des sourciers et des contestations extraordinaires, certains Pères jésuites faisant des livres disant que par un effet physique, les petites particules qui s'evaporent de l'eau rentrent dans la baguetle et la font tourner; d'autres Pères jésuites disant: «Non, c'est bien trop dangereux, on ne peut pas juger de choses morales avec la baguette, le diable est dans la baguette. Surtout ne touchez pas une baguette, vous risquez d'ètre damnés... »

Il existe un décret de l'Inquisition de 1701 qui interdit l'emploi de la baguette comme témoignage en justice.

Personnellement, je suis partisan du signal du sourcier qui existe physiquement dans beaucoup de circonstances; je suis aussi partisan de ce décret et ne désire nullement qu’il soit rapporté...

On trouve, à la campagne, des puisatiers, sourciers experts au point de vue des signaux, et qui n'hésitent pas à prendre la responsabilité de creuser des puits à leurs frais et de n'être payés qu'après. Cette attitude inspire un certain respect et l'on est obligé de les prendre au sérieux; d'ail- leurs, il n'est pas facile de discuter avec ces genslà, parce qu'ils manquent d'entraìnement scientifique, ont souvent une mentalité particulière, beaucoup d'assurance, et se sont forgé un système d'explication intenable : il est rare que l'on puisse pousser très loin et se baser beaucoup sur l'expérience des autres. Mais, étant donné que je ressens les signaux sans prétendre à aucune performance, en parlant seulement de ce que je suis capable de sentir, je pense que je peux tout de même risquer d'approcher un peu le problème.

On trouve aussi des gens très simples (c'est même une majorité) qui disent : avec moi, cela marche. Ils coupent une branche de noisetier dans un buisson, ils arrivent et disent : voyez, j'ai un signal ici, mais je ne sais pas pourquoi. Autrement dit, ils n'ont aucune interprétation. J'en ai vu opérer, j'ai cherché à en faire autant, mais j'avais déjà des idées a priori, ce n'est peut-être pas la meilleure attitude.

«J'emporte une baguette, j'arrive sur une flaque d'eau : rien; sur une rivière : rien; sur un ruisseau qui coule: pas davantage; sur un étang avec un petit pont: encore moins. Bref, c'est dommage, mais je ne suis pas sourcier. Voila ce que presque tout le monde déclare.

Une fois tout de même, j'étais sur un terrain où je pensais que j'avais peut-être des signaux. Je me fait alors pousser dans une voiture automobile, une $2 \mathrm{CV}$ (dont vous connaissez l'agrément et la qualité de la suspension), en me promenant à la vitesse d'un homme au pas, et je retrouve mes signaux.

Je me dis : ce n'est pas possible que ce soit le champ électrique; je suis dans une petite cage de Faraday; il n'est pas possible que ce soient des ions issus de l'eau qui attaquent mon odorat, etc., puisque je suis dans une boìte à peu près fermée, il n'y a plus que le champ magnétique. Autrement dit, avec ma position personnelle de physicien, je n'ai pas douté un seul instant qu'un intermédiaire physique ne soit nécessaire au déclenchement du réflexe.

Mais je dois vous dire que la corporation des radiesthésistes ne se laisse pas apprivoiser si facilement et qu'elle ne se sent absolument pas liée, en général, par cette considération. Je m'en tiens simplement à ma position, avec cette idée que le magnétisme joute un rôle. Mais une voiture est elle-même un petit tas de ferraille rendu magnétique par le champ terrestre, elle a un moment magnétique. Une voiture automobile cause une perturbation de $1 \gamma$ $\left(10^{-5}\right.$ gauss) à $15 \mathrm{~m}$; on en déduit, par la formule $\mathrm{M} / \mathrm{R}^{3}$ la valeur du moment magnétique équivalent. Il est facile d'empoigner une baguette et de passer devant une voiture: on constate que l'on a un signal et on constate aussi certaine particularité : si l'on se promène deux ou trois fois plus lentement, on réagit beaucoup moins bien, on peut même perdre toute espèce de signal.

Ceci suggère que le sourcier aurait son réflexe déclenché au moment où il passe dans une inégalité magnétique. Le terme «gradient» ne s'applique pas à la variation d'un vecteur. Pourtant, je vais dire qu'on passe daus un gradient parce que c'est bien commode, et vous me pardonnerez pour cette fois.

Le gradient, pour cette occasion, c'est la région 
où le champ magnétique n'est plus tout à fait le champ terrestre et subit une perturbation sur une distance de quelques mètres. Si l'on suit ces idées, on peut s'occuper d'abord de vérifier par des expériences plus ou moins soignées, plus ou moins sérieuses, la réalité du déclenchement magnétique et l'on peut essayer de se poser le problème de l'eau.

Le somrcier n'aurait-il pas, de temps en temps, des signaux authentiques sur l'eau par l'intermédiaire du magnétisme? La réponse est la suivante : l'eau qui coule dans une rivière n'a rien de magnétique, l'eau qui dort dans un étang non plus; mais l'eau qui filtre dans des milieux poreux produit des courants et des potentiels d'électrofiltration qui sont à la rigueur capables de faire circuler des courants.

Imaginons une source dont l'eau jaillit et que l'électrofiltration ait lieu sur un certain trajet dans le sol; les potentiels qui se développent vont du signe moins en amont au signe plus en aval. Le milieu, la terre, est conducteur et ces potentiels ferment des lignes de courant qui peuvent créer un champ magnétique. Il est possible, après tout, qu'on ait suffisamment de gradient pour obtenir une détection.

Ceci conduit à faire des expériences avec des objets dont on peut grossièrement évaluer le champ magnétique. On se rend compte alors que la sensibilité est totale pour des valeurs curieusement assez faibles, 0,3 ou $0,5 \mathrm{mG} / \mathrm{m}\left(10^{-3}\right.$ gauss $\left./ \mathrm{m}\right)$. Des champs artificiels réglables deviennent alors nécessaires. Une des premières choses que nous avons constatées quand nous avons voulu faire des champs artificiels, c'est une curieuse saturation. Quand on parcourt un gradient vingt fois trop fort, on n'obtient jamais ce qu'un sourcier détecte avec sûreté. Si l'on peut parler d'un seuil, il serait plutôt vers 0,2 ou $0,3 \mathrm{mG} / \mathbf{m}$; des gradients très forts de 20 on $30 \mathrm{mG} / \mathrm{m}$ vous saturent et vous restez parfois pendant plus d'une minute, en sortant d'un champ trop fort, avant d'être à nouveau capable de jouer au sourcier.

Voilà la situation, aussi bizarre qu'elle puisse paraître, au point de vue magnétique.

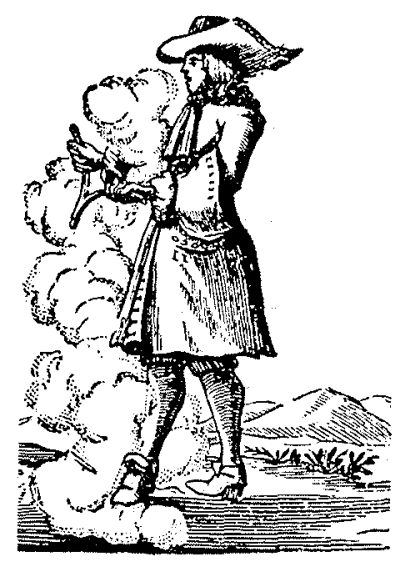

Je reviens à l'eau. Je voudrais bien, dans la mesure où j'apporte quelque support aux sourciers, que l'eau crée une distribution de courant électrique capable de produire des anomalies magnétiques de l'ordre du mG, peut-être un peu moins, sur quelques mètres. Finalement, ce n'est pas tellement facile.
Premiere remarque: tune distribution de courants avec une symetrie de révolution ne donne aucun champ maǵnétique à l'extérieur. Une boucle, au contraire, est équivalente à un aimant. Donc le sourcier peut mancruer une bonne source qui créerait des courants bien symétriques et détecter une source insignifiante qui serait caractérisée par une bonne dissymétrie.

Denxieme remarque: il faut qu'il y ait assez de courant. Or, ce n'est pas facile. Si l'on imagine dans la terre une distribution de courant par filets parallèles dans la terre, si l'on admet des valeurs de l'ordre de $50 \mathrm{~mA}$ par $\mathrm{m}^{2}$ et si on limite à un plan vertical cette distribution de courant, on a alors un gradient de champ magnétique détectable. Ces $50 \mathrm{~mA}$ par $\mathrm{m}^{2}$ exigent des forces électromotrices dans les environs et une conductivité suffisamment grandes toutes les deux.

Dans les livres de prospection géophysique, on cite des conductivités des roches qui sont quelconques et qui augmentent énormément si la roche est saturée d'eau. Sans discuter ici de chiffres, disons que seuls les cas les plus favorables conduiront assez de courant.

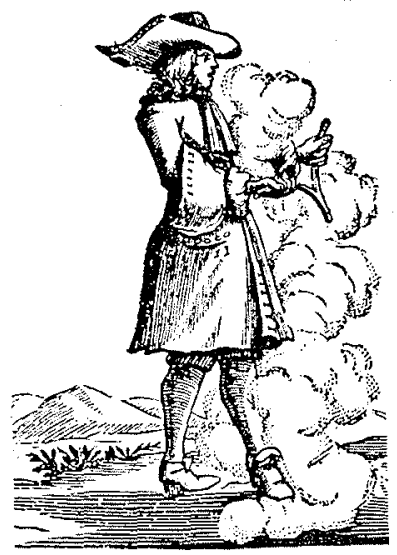

Voyons maintenant les f.e.m. : que donne l'électrofiltration? Il est très facile de faire des expériences sur l'électrofiltration : il suffit de disposer d'un voltmètre électronique à haute impédance, comme on en trouve maintenant, et qui ne courtcircuite pas les phénomènes. Si j’ai, par exemple, un talus avec des fuites, séparant un niveau d'eau d'un fossé avec $1 \mathrm{mi}$ de différence de hauteur, si je jette d'un côté el de l'autre deux électrodes impolarisables, ou seulement si je mesure assez vite, je trouve $50 \mathrm{mV} / \mathrm{m}$, ce qui est un ordre de grandeur de force électromotrice d'électrofiltration tout à fait acceptable, caractérisant la hauteur d'eau de $1 \mathrm{~m}$.

Je vais trahir un petit secret : nous faisons éclater des bombes atomiques dans une montagne de granit au Sahara; il y a done des galeries horizontales dans cette montagne; de temps en temps, on $y$ trouve des failles où l'eau coule et le granit y est délité; on a une sorte de bouillie au lieu d'avoir de la roche solide. Si je place une boule près du sol de la galerie et une autre à $2 \mathrm{~m}$ au-dessus, j'observe $80 \mathrm{mV}$ de différence de potentiel : c'est le même ordre de grandeur. On ne voit vraiment pas autre chose que l'électrofiltration pour créer ces différences de potentiel.

Or, les f.e.m. d'électrofiltration restent faibles 


\section{Y. ROCARD}

pour expliquer les signaux : dans mon livre $\left(^{*}\right)$, on trouve la description d'un petit barrage qui a $70 \mathrm{~cm}$, peut-être $1 \mathrm{~m}$ de longueur, avec $30 \mathrm{~cm}$ de différence de hauteur d'eau. On mesure des différences de potentiel assez impressionnantes si l'on met des petits piquets dans la terre à côté, mais elles ne signifient pas grand-chose quant aux courants qui peuvent passer. Si l'on arrive avec une baguette, il est impossible de rien détecter en ce site, bien que ce soit une très bonne petite source qui devrait très bien marcher.

Par contre, dans un autre site qui est aussj décrit dans mon livre, si je me promène sur le talus qui a des fuites, l'échelle change brusquement: si je pique des piquets dans la terre, je trouve 200 et $400 \mathrm{mV}$ de différence de potentiel très facilement, et là j'ai des signaux de baguette. Mais ce talus est un talus artificiel de pierre, et on l'a rendu étanche avec de l'argile; par conséquent, je ne suis déjà plus dans le domaine de l'électrofiltration, j'ai les forces électromotrices de l'argile qui viennent en rajouter : en gros, la f.e.m. d'une pile de concentration donnant des potentiels peut-être dix fois plus forts que l'électrofiltration. Dans ces conditions, on a des signaux qu'il est à la rigueur possible d'attribuer à l'eau.

Les signaux peuvent être différents suivant que l'on est l'été ou l'hiver, si le régime des eaux change. On ne détecte pas de la même manière suivant le temps, suivant la baisse générale des eaux dans le pays. Ceci conduit à penser que les sourciers atteignent tout de même ces petits effets. Par contre, quand on vous dit : l'eau est à $150 \mathrm{~m}$ de profondeur l'été et à $90 \mathrm{~m}$ l'hiver, il n'y a pas de rapport avec le système d'explication que je donne, et il ne m'est pas possible d'attribuer une confiance totale aux sourciers qui prétendraient à de telles performances. Je ne leur reconnais même pas la possibilité d'avoir des idées sérieuses sur le débit, parce que très peu d'eau qui filtre dans un milieu très serré fabriquera autant d'occasions de différences de potentiel et aussi de conductivité que beaucoup d'eau qui filtre, comme un torrent, à travers un tas de pierres.

La profondeur? On peut admettre que si le sourcier se promène et s'il a des signaux avant et aprés un point, il définit ainsi la base d'un triangle au sommet inférieur duquel il peut y avoir la cause du signal; cela donne une idée qualitative de la profondeur, qui peut être confirmée par vingt-cinq ans d'expérience dans un pays donné, où l'homme connaît tous ses puits. On peut admettre que le sourcier donne une indication qui peut être valable sur la profondeur.

Je n'ai guère davantage à dire sur l'eau. Je crois que les sourciers honnêtes ont souvent des signaux causés par l'eau, mais je les vois peu utilisables, pour les besoins d'envergure industrielle notamment. Néanmoins, dans des pays favorables, on aimera mieux les indications d'un sourcier que rien du tout pour savoir où creuser un puits dans la nappe phréatique que l'on a sous les pieds.

Imaginons, par exemple, un pays de granit, où le granit ait des hauts et des bas et où l'humus ait une épaisseur de $3 \mathrm{~m}$ environ; si l'on creuse sur un

* Le signal du sourcier, par Y. Rocaro, Dunod, éditeur, Paris, $2^{\circ}$ ed. 1964. sommet de granit, ce n'est pas si bien que si l'on creuse dans une rigole, il vaut mieux ne pas se tromper, et dans ce cas, le signal du sourcier nous apporte une aide acceptable.

Mais je voudrais revenir un peu en arrière pour m'étendre davantage sur l'aspect magnétique de l'affaire.

Les gens qui sont contre et surtout ceux qui ont souffert des sourciers dans des opérations financières, ou alors qui attendent trop de précisions des sourciers, émettent un doute total sur la réalité même du signal et disent: pas d'effet physique, autosuggestion totale, illusion complète d'un bout à l'autre.

Je suis physicien et non physiologiste; j'avoue avoir été déconcerté au moment où ces objections m'ont été présentées.

Cependant, je suis contre l'idée d'une autosuggestion totale, notamment pour la raison suivante : il y a une hystérésis considérable dans le signal.

Par exemple, ici dans cette salle, on est très sollicité dans tous les sens; les signaux que je pourrais avoir, je suis incapable de les attribuer à un objet précis, sauf le micro ici présent sur son pied, qui a un petit moment magnétique dans le champ terrestre et qui me donne certainement $1 \mathrm{mG}$ à $1 \mathrm{~m}$. A part cette exception, je ne suis pas à l'aise pour localiser l'origine d'un signal, mais j'ai l'impression que si je prends ma baguette et que je me place là, je peux toujours essayer : je me dome d'abord plusieurs secondes pour avoir mes muscles à peu près en état et je marche... Voilà le signnal déclenché, je l'attribue plutôt à cela qu'à autre chose et j'admets qu'il a fallu $2 \mathrm{~s}$ pour le retarder; si j'étais autosuggestionné par la présence de cet objet (le micro), il me semble que je serais déclenché tout de suite. Mon cerveau fonctionne bien plus vite que mes réflexes de sourcier.

Ce temps d'hystérésis fait que j'ai peine à croire à beaucoup d'intervention du cerveau. Cependant, on fait un geste, c'est donc que l'on veut détecter; on marche, c'est donc que l'on veut aller là. Il y a tout de même intervention de la volonté, mais, curieusement, le cerveau n'est pas informé que quelque chose s'est passé dans les muscles et la baguette file des mains sans que la volonté y contribue.

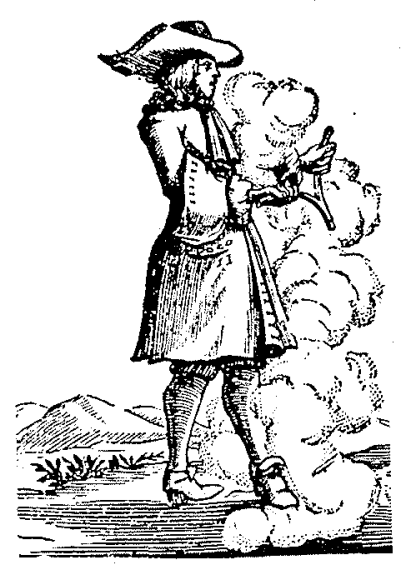

Il n'y a aucun mystère du còté de l'énergie. La baguette est élastique, e'est même sa seule propriété importante à considérer. Cette baguette, on la déforme, on lui donne de l'énergie potentielle et cela suffit pour tout le reste de l'affaire : un simple 
affaiblissement de la prise libère son mouvement. L'homme n'a pas à fournir d'énergie, il a simplement à être surpris et à lâcher un peu, et il est surpris indépendamment de sa volonté. C'est ce manque d'intervention de la volonté qui rend la sensation si surprenante.

On peut imaginer que quelque chose se détraque dans le système nerveux végétatif, celui qui échappe à l'action du cerveau, qui fait digérer, qui vous maintient debout, etc. On peut aussi imaginer simplement une baisse du tonus musculaire qui serait due à l'action de ce champ magnétique.

Si, par exemple, je suis capable, en serrant un dynamomètre, d'exercer une force de $30 \mathrm{~kg}$, il est possible, en m'engageant dans un endroit où je subis une action physique, que je ne puisse plus exercer que 29 ou $28 \mathrm{~kg}$; comme je me suis mis dans un état dit instable, après tout cela suffit pour que la baguette s'en aille. Je suis à peu près convaincu qu'il n'y a pas à chercher plus loin et que le phénomène peut se réduire à cette diminution du tonus.

Lidée que le gradient magnétique, ou la petite inégalité magnétique, joue un rôle, soulève immédiatement une foule d'objections :

D'abord, 0,5 gauss, qui est le champ terrestre, ce n'est pas beaucoup; $1 / 1000^{\mathrm{e}}$ de gauss, c'est encore bien moins; une action de $1 / 1000^{\circ}$ de gauss sur le moment magnétique d'une molécule donne des énergies beaucoup plus petites que $\mathrm{K} T$, la valeur moyenne de l'énergie des molécules dans l'agitation thermique $\left(^{*}\right)$. Comment admettre une excitation aussi faible?

Considérons cependant la résonance magnétique nucléaire : elle permet de mesurer en faisant tourner par exemple les protons d'un litre d'eau, des champs magnétiques avec des précisions qui vont de $10^{-6} \dot{a} 10^{-7}$ gauss. Nous avons done la preuve que des énergies bien inférieures à KT peuvent être accessibles à des mesures, pourvu que suffisamment d'effets moléculaires se groupent pour un résultat macroscopique.

Autre remarque : on peut envisager dans le corps humain des circuits qui seraient électriques et on pourrait se demander si la force électromotrice d'induction due à la variation de flux, due au fait qu'on passe dans des champs aussi faibles, peut induire un signal? Non, les valeurs sont beaucoup trop faibles. Je suis tout à fait convaincu que la force électromotrice, dans le corps humain, ne peut produire la réaction sourcière. Par contre, la résonance magnétique nucléaire, que nous venons de mentionner fournit une possibilité :

Si je prends un proton, le noyau d'un atome d'hydrogène, c'est un petit aimant d'une part, et d'autre part un petit gyroscope, le champ magnétique lui applique un couple, et ce proton précessionne à une vitesse qui se trouve être d'environ $2000 \mathrm{tr} / \mathrm{s}$ dans le champ magnétique terrestre. Il $\mathrm{y}$ a beaucoup de protons dans le corps humain et aussi des atomes de carbone, des métaux dans les os, etc. Si on vit dans un champ uniforme, tous les protons tournent ensemble, ils sont tous bien à la même vitesse et peuvent avoir des rela-

* $K=1,38.10^{-16}$ si $T$ est en degrés absolus et si cette énergic est mesurée en ergs. tions de phase constantes; si, par hasard, ils sont dans des molécules organiques, il $y$ a ainsi des structures qui se conservent. Si, au contraire, on avance dans un endroit où le champ est modifié de $0,5 \mathrm{mG}$, cela veut dire qu'il y a $2000 \mathrm{tr}$ en un point et 2001 ou $2002 \mathrm{tr}$ à $1 \mathrm{~m}$. A ce moment, des effets de battement lents à la frécuence cqui est la différence, soit ici $1 \mathrm{tr}$ par seconde, se produisent. Or, la circulation du sang va faire passer des protons mobiles devant des protons fixes, et ces battements à basse fréquence peuvent alors donner des effets importants dans les tissus du corps humain.

Si l'on admet de se laisser entraîner dans ce système d'explication, cela marche assez bien, parce que l'hystérésis est expliquée, elle est en effet de l'ordre de grandeur des temps de relaxation de la résonance magnétique nucléaire : on voit, dans les expériences de résonance magnétique nucléaire, qu'il s'écoule plusieurs secondes avant qu'un signal de résonance prenne sa forme sur un oscillographe, et on voit sur le terrain que le sourcier, en s'écartant de son point sensible, a besoin aussi d'un temps non négligeable pour être actionné. Mettonsnous maintenant dans un gradient trente fois trop fort, nous aurons des battements à une fréquence beaucoup trop rapide, qui, visiblement n'aura plus le même effet physiologique. C'est surtout le fait de la saturation qui rend intéressante la possibilité d'explication par un battement. Mais je m'arrête là, je n'ai pas été capable de préciser davantage. En tout cas, la physique offrirait ces possibilités.

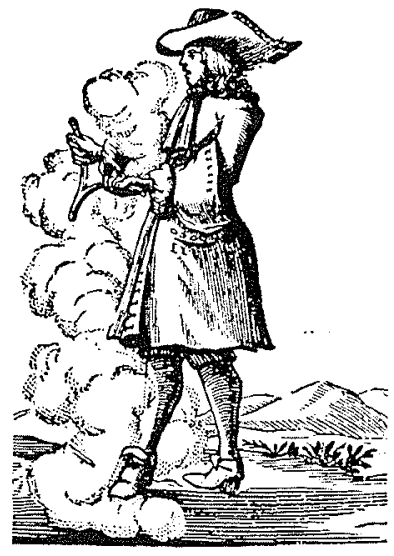

On trouve dans le commerce des magnétomètres a protons, portatifs; il est vrai qu'on les paye de 15000 à 50000 francs; néanmoins on les trouve et on peut donc se promener et faire des mesures magnétiques. Par conséquent, partout où un sourcier officie, on peut immédiatement vérifier la situation, et on peut faire l'inver'se, c'est-à-dire reconnaitre des points et $\mathrm{y}$ envoyer un sourcier. Dans lous les cas de ma connaissance, par exemple un chemin de terre sur $800 \mathrm{~m}$ ou $1 \mathrm{~km}$, si l'on dit à un sourcier de mettre des marques là où il a ses signaux, qu'il y laisse tomber un petit caillou blanc et si l'on arrive avec un magnétomètre, on trouve chaque fois des variations. Quand on trouve 0,3 , $0,4 \mathrm{mG}$ sur $1 \mathrm{~m}$, il est tentant de relier ces milligauss à l'eau; quand on trouve 2 ou $3 \mathrm{mG}$ sur $1 \mathrm{~m}$, c'est déjà trop pour l'eau : je reviendrai sur cet aspect des leurres qui font que le sourcier se trompe souvent.

La situation inverse où l'on envoie le sourcier là où on a mesuré une anomalie magnétique, marche 
à tout coup, les objets les plus vulgaires donnent des signaux, une simple espagnolette de fenêtre déclenche son sourcier à $1 \mathrm{~m}$. Mais venons-en maintenant à des expériences prouvant qu'il y a un effet physique indépendant de l'autosuggestion.

On peut faire des champs avec des cadres, ces cadres auront commodément $50 \mathrm{~cm}$ sur $1 \mathrm{~m}$, pour une centaine de spires de fil fin, dans lesquelles on fait passer des $m A$ qui donnent des $m G$ au centre du cadre et des dixièmes de mG en passant devant.

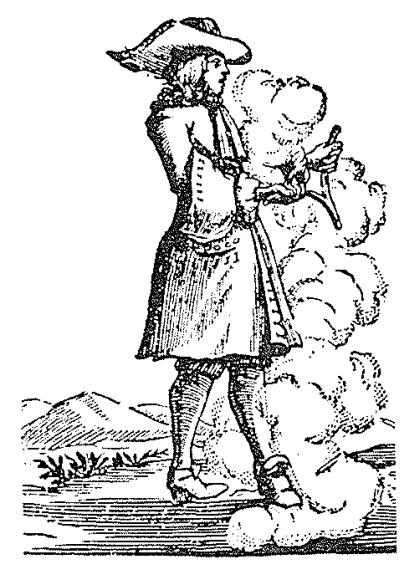

Imaginons le cadre posé sur un chevalet et l'homme passant devant. On cherche un site amagnétique, sans gradient. Cela se trouve, bien que ce ne soit pas très facile, si on se met n'importe où, c'est bien rare qu'on n'ait pas quelque signal qui déclenche sur une longueur de 10 ou $15 \mathrm{~m}$. Exigeons d'avoir 10 ou $15 \mathrm{~m}$ pour que l'homme ait pris un certain équilibre. On opère alors ainsi : on le fait passer devant le cadre en lui disant que le courant est mis; il doit détecter. Evidemment, quand il passe devant, il sait que c'est là et il détecte, mais il a pesé sa sensation.

Denxième temps: on fait repasser le sourcier devant le cadre avec courant nul et on le prévient; il doit reconnaître qu'il ne détecte pas. Quoi qu'il en soit, on lui donne cette facilité. Ensuite, on le fait repasser sans lui dire s'il y a du courant ou non. On lui a beaucoup facilité les choses : il sait où il doit détecter et il sait à peu près le moment, on lui demande simplement de décider s'il a un champ magnétique ou s'il n'en a pas, et en outre c'est un champ qu'il a détecté une minute avant.

Si l'on opère ainsi pour l'ensemble de cette expérience, le sourcier ne se trompe jamais, c'est-à-dire que des séries de 5 ou 6 expériences semblables donnent 5 ou 6 résultats tous bons.

Mais je tiens assez à ce que le sourcier fasse un aller et retour, de manière qu'il détecte par sa gauche et par sa droite. Cela fait déjà 30 ou 36 passages; on en a vite assez et il ne faut pas éterniser ce genre de séries. Néanmoins, dans la même journée, on peut bien en faire 2 ou 3 .

On peut aussi varier un peu, par exemple on fait une série de coups inconnus, mais, chaque fois que le sourcier est passé, on lui dit s'il a raison ou tort, ainsi peut-il se «peser».

En opérant de cette manière, nous avons aussi obtenu $100 \%$ de succès sur des séries de 8 ou 10 en prévenant le sourcier qu'il opérait bien et en lui donnant le courant optimal. Si l'on donne un courant trop faible, on le fait se tromper à peu près comme on veut. Si on lui met un courant trop fort, il y a la saturation que je signalais et à laquelle j'attache beaucoup d'importance, parce qu'elle joue certainement un rôle considérable dans l'interprétation du mécanisme, et, d'autre part, si on n'en tient pas compte dans les expériences, on porte alors un jugement très faux. Le courant trop fort, on le détecterait plus loin en allant plus vite, parcourant ainsi un gradient plus faible qu'on serait allé chercher plus loin. Mais si l'on est astreint à une technique de passage, on est limité à ce point de vue et aussi dans la valeur des champs. La limitation est dans un rapport de 1 à 10,1 à 20 peutètre, il faut néanmoins en tenir compte.

Voilà la situation en ce qui concerne la réalité du phénomène. Nous sommes assez nombreux à avoir fait ces expériences : l'Ecole Normale Supérieure est devenue, si j'ose dire, un repaire de sourciers : dans une promotion d'élèves, il y en a $75 \%$ qui réagissent instantanément à la baguette, et on trouve aisément deux ou trois sujets qui se révèlent très « sensibles».

La possibilité de détecter l'eau me paraît donc acquise, avec cette limitation qu'il n'est pas facile d'avoir des champs magnétiques suffisants.

Il reste une possibilité : c'est que d'autres effets que le magnétisme déclenchent le sourcier. Là, je n'ai rien à dire; ayant trouvé une possibilité du côté du magnétisme, j'ai déployé des efforts pour que l'eau fournisse l'effet magnétique. S'il y a d'autres possibilités, j'aimerais les connaître, mais jusqu'ici je n'en vois pas.

Voici encore quelques détails. On détecte en voiture, on détecte en avion, mais il ne faut tout de même pas se laisser trop impressionner. Vous ètes dans un avion et vous vous déplacez 100 fois ou 200 fois plus vite qu'à pied, suivant que vous êtes dans un «Constellation» ou dans une « Caravelle $»$. A ce moment-là, le gradient est de $0,3 \mathrm{mG}$ par mètre, vous ne le détectez pas, vous passez beaucoup trop vite dessus; mais un gradient de $0,3 \mathrm{mG}$ sur $200 \mathrm{~m}$ que vous parcourez à une vitesse 200 fois plus grande, vous fait subir des variations dans le temps du même calibre que $0,3 \mathrm{mG}$ sur $1 \mathrm{~m}$ parcouru à pied.

J'ai noté sur certains trajets au Sahara quelques rares signaux de cette espèce, probablement attribuables à quelque colline un peu magnétique, plutôt qu’à un immense fleuve souterrain. Si on considère la performance du sourcier par rapport à celle du magnétomètre aéroporté, l'appareil est tout de même 50 fois supérieur. Mais les gros accidents des enregistrements magnétiques ramenés par les avions sont accessibles aux sourciers; or, la baguette est tout de même assez simple, assez facile à manier; il n'est pas absolument ridicule de faire ainsi une première exploration. On peut toujours la tenter, mais cela exige un vol absolument rectiligne et sans aucune accélération, parce que tout dérangement physiologique est susceptible de donner un signal (je ne dis pas que tout dérangement actionne le sourcier, mais il peut donner un signal).

Je peux d'ailleurs en donner un exemple : lors d'une explosion nucléaire souterraine, des disciples à moi se sont tous placés avec une baguette au «count down $» 10 \mathrm{~s}, 9 \mathrm{~s}, \ldots$ etc. et zéro, ils n'ont rien obtenu, ils étaient à une quinzaine de kilomètres, mais 2 ou $3 \mathrm{~s}$ après, quand l'onde séismique 
leur est passée sous les pieds (on la sent), toutes les baguettes sont parties (légèrement d'ailleurs).

Autrement dit, une petite vibration, ou une perte d'équilibre, donne cette baisse du tonus musculaire qui suffit à la baguette pour s'en aller.

Je reviens à l'avion : je dois refuser tout signal où l'avion remue, où je sens une accélération. D'autre part, j'ai des possibilités nouvelles par les masses magnétiques que l'avion comporte autour de moi, et si l'avion change son cap avant d'atterrir, lorientation des masses dans l'avion est modifiée, le champ magnétique auquel je suis soumis subit ces petites perturbations : on a des signaux de cette espece, on peut les accepter ou les refuser suivant qu'on avoue qu'on n'a pas été dérangé physiologiquement ou qu'on l'a été, mais en tout cas, c'est possible: un sourcier assis dans la queue d'une Caravelle perçoit le train d'atterrissage qui se rentre.

J'insiste beaucoup sur le fait que l'homme n'est pas une boussole, le sourcier pas davantage; il n'a aucune indication de direction; néanmoins, on a une indication de sens. Autrement dit, si l'on détecte quelque chose, il n'est pas indifférent de marcher dans un sens ou de marcher dans l'autre, ce que j'attribue tout simplement au fait que le champ magnétique dans lequel je suis est plus faible d'un côté que de l'autre et qu'il y a quelque chose de dissymétrique dans le corps humain; ceci est acceptable.

Si l'on incline vers l'explication qui ferait intervenir la résonance magnétique nucléaire, c'est satisfaisant, parce que la résonance magnétique nucléaire ne met en jeu que la valeur totale du champ. Mais si je veux devenir une boussole, qu'est-ce que je fais? j'empoigne une tige de fer doux, je la mets dans ma manche, je me place ainsi... et je suis actionné par un champ produit par mon fer doux et qui change un peu suivant la direction par rapport au nord et au sud.

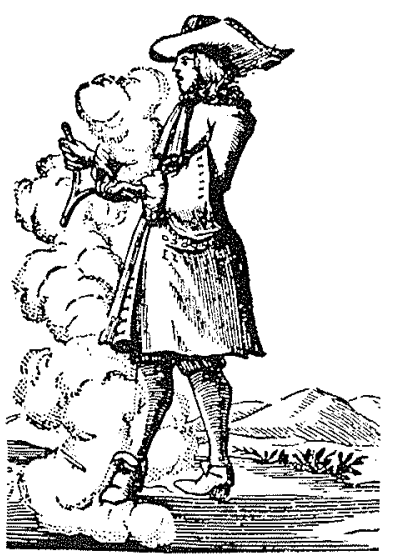

Par conséquent, il est possible de détecter quelque chose, une orientation, mais, même si l'on tient compte de l'hystérésis du sourcier et de l'incertitude du caractère qualitatif des perceptions, on n'est pas une boussole très bonne; néanmoins, on a des impressions de direction. Faire un quart de tour avec le nord n'est pas la même chose que faire un quart de tour avec l'est en face de soi.

Une dernière expérience: je tourne sur mes pieds à la vitesse qui m'est possible, c'est-à-dire un tour toutes les $2 \mathrm{~s}$, et comme vous voyez mon signal se déclenche très vite (un tour); si je tournais indé- finiment, j'aurais une perte d'équilibre, mes canaux semi-circulaires seraient troublés, etc., donc, il est possible que l'effet soit purement physiologique. Si toutefois il est magnétique, il $y$ a une équation :

$$
\frac{\Delta \omega}{\omega}=\frac{\Delta \mathrm{H}}{\mathrm{H}}
$$

qu'il faul lire ainsi :

Un changement de la vitesse de précession des protons, qui serait $\Delta \omega$ et équivalant à un changement du champ magnétique, qui serait $\Delta \mathrm{H}$. Or, $\omega$, c'est $2000 \mathrm{tr} / \mathrm{s}$, et $\Delta \omega$, c'est ma vitesse de rotation, $\mathrm{tr} / \mathrm{s}$; ceci donne $\Delta \mathrm{H}=(\mathrm{H} / 4000)$ soit $0,12 \mathrm{mG}$; c'est bien l'ordre de grandeur du seuil de sensibilité que nous reconnaissons au sourcier.

Toutes ces petites expériences sont suspectes. Je ne voudrais pas vous inspirer trop de confiance, mais chacun de vous peut les répéter. Dans une assemblée comme la vôtre, Messieurs, il y a $75 \%$ de sourciers (ce qui est déjà très honorable), dont $50 \%$ sont meilleurs que moi et dont 2 ou 3 auraient de bien meilleures performances.

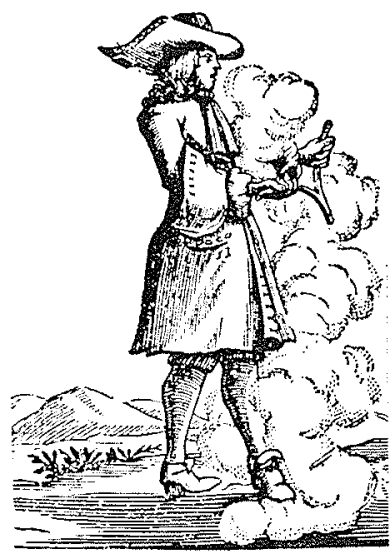

Quelle est la différence entre le bon et le mauvais sourcier? Ils ressentent tous à peu près la même chose. Aucun (même les très bons) ne détecte avec sûreté notablement au-dessous de $0,1 \mathrm{mG}$, il suffit d'en prendre un et de l'obliger à essayer. Mais le bon sourcier a des réflexes beaucoup plus franes, plus décidés, et arrive beaucoup plus vite à un résultat. La différence est dans la qualité du réflexe.

C'est comme pour la vue : pour voir, il faut qu'il $\mathrm{y}$ ait de la lumière, si le jour baisse, on ne voit plus rien. La différence entre une bonne vue et une mauvaise vue, chacun la connaît, elle existe et pourtant l'éclairage est le même qui permet la bonne et la mauvaise vues. Pour les sourciers, c'est à peu près pareil : cause physique uniforme, performance physiologique variable.

Mais je ne puis terminer cet exposé sans mentionner les cas où le sourcier a, sur le terrain, des signaux non attribuables à l'eau. En éliminant toutes occasions de déséquilibre physiologique dans la marche sur un mauvais terrain, il reste à considérer les leurres magnétiques.

Un Gaulois, fuyant les légions de Labienus a laissé choir son épée en fer : voilà la terre rendue magnétique pour vingt siècles. Un vieux soc de charrue cn fera autant.

Un éclair qui frappe la terre peut, selon la constitution des roches et même de l'humus, v laisser un moment magnétique dû au passage d'un fort courant, 50000 à $500000 \mathrm{~A}$. 
L'humus lui-même contient normalement de l'oxyde de fer $\mathrm{Fe}_{2} \mathrm{O}_{3}$ non magnétique; mais s'il est réduit par des détritus organiques, par un feu de branchages, etc., on lui trouve une susceptibilité magnétique de l'ordre de $1 / 1000^{\circ}$, plutôt un peu moins. La frontière d'un bane d'humus ainsi modifié donne un signal sourcier, et à $1 \mathrm{~m}$ du sol on $\mathrm{y}$ mesure bien le gradient de $0,3 \mathrm{mG}$ sur $1 \mathrm{~m}$, assez fréquemment rencontré.

Par contre, dans la propriété où sont l'étang et le talus que j'ai évoqués plus haut, nous trouvons un signal sourcier net et très localisé. Le magnétomètre $\mathrm{y}$ mesure entre 2 et $3 \mathrm{mG}$ par mètre selon la direction.

Le signal est très stable, mais, trop fort, il me semble impossible qu'il soit dû à l'eau. Un obus enterré de la guerre de 1870 expliquerait tout. Nous sommes en effet sur le site de la bataille de Coulmiers. Les obus dressés qui ornent le perron des mairies jouissent de la même propriété sourcière.

Bref, attention au leurre magnétique.

Voilà, Monsieur le Président, Mesdames, Messieurs, ce que j'avais à vous dire : un effet physique peut déclencher le sourcier sur le terrain. Un léger gradient magnétique constitue un tel effet. Il n'est pas exclu, mais pas du tout établi, que d'autres causes puissent le faire aussi. La détection de l'eau qui filtre, souvent revendiquée par l'homme de l'art, peut se relier à cet effet magnétique.

Si j'ai oublié certains aspects du problème, je suis à votre disposition pour répondre à vos questions.

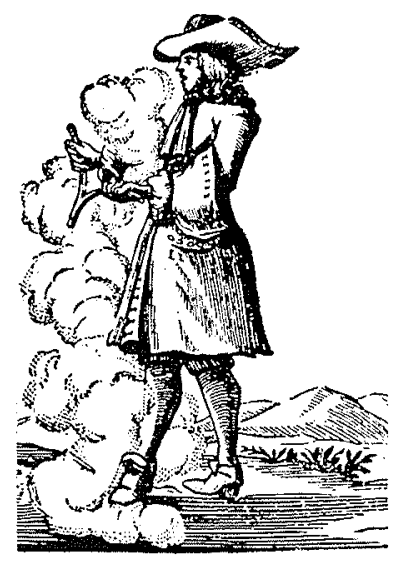

Discussion.

Président: M. Chapouthier.

M. le Président remercie M. le professeur Rocard et avoue avoir été impressionné par le nombre et la qualité des experiences auxquelles il a fait allusion.

M. le Président se demande, toutefois, si, à force de teuir la baguette en la tendant légèrement, il n'y a pas un affaiblissement du tonus musculaire de l'expérimentateur qui se traduirait pour lui par un déclenchement du signal, sans aucun rapport avec une réalité physique.

M. Rocand reconnaît un rôle à la fatigue mais ne croit pas à l'absence de réalité physique aans les expérimentations radiesthésiques, car le magnétomètre confirme la présence d'un champ qui, dans un certain nombre de cas, peut être considéré en toute objectivité comme produit par l'eau, en de nombreux points où les sourciers ont réagi. Depuis des années, des physiciens allemands ont énumeré de tels cas d'expériences. M. Rocand s'est appliqué plutôt à faire le passage entre le magnétisme et l'eau en raison de sa spécialisation professionnelle dans les puits de pétrole, les sondages, l'électrofiltration et le self-potentiel de l'argile.

M. le Président ajoute que M. Rocano a pu, ainsi, limitev de façon importante le domaine de validité de l'expérience du sourcier au cas de la création d'un champ magnétique par l'eau.

M. Fock demande :

$1^{\circ}$ si le sourcier est sensible seulement au gradient du champ ou à la vitesse avec laquelle varie ce champ (par suite du déplacement de l'observateur) dans la boucle formée par la baguette, les bras et le corps;

$2^{\circ}$ ce qui arrive si l'opérateur s'oppose à la chute de la baguette (il avait vu, en pareil cas, un sourcier briser sa baguette).

M. Rocard confirme l'action de la vitesse, nécessaire pour la plupart des sujets. Il signale que :

a) très souvent, au-dessus du sol, on observe un petit gradient vertical du champ magnnétique, qui diminue de 0,2 à 0,3 milligauss si on s'élève de 20 à $30 \mathrm{~cm}$. Le sour- cier, dans sa marche horizontale, ne semble pas pereevoir ce gradient, à la hauteur de ses mollets et qui ne varie pas;

b) dans beaucoup de cas, on manque un signal si on se déplace trop lentement; on l'obtient si on marche deux fois plus vite.

M. Rocard précise encore, à l'occasion de la question de M. Foch, que la résonance magnétique mentionnée dans son exposé provoque la rotation des protons à 2000 tours et que le cœur et la circulation du sang font passer les uns devant les autres des protons qui, dans le cas d'un gradient, ont des petites différences de vitesse de rotation. Compte tenu des temps d'hystérésis qu'on appelle temps de relaxation, e'est par le procédé de la résonance nucléaire que l'on a, à très petite distance, des possibilités de battements dans le corps humain. On en déduit que la simple position dans un gradient sans vitesse devrait suffire pour obtenir la détection, et c'est bien le cas pour certaines personnes très douées. Il a des gens qui, tenant la baguette, se mettent à trembler et la lâchent sans bouger. On peut aussi mentionner le pendule, avec lequel on détecte immobile, mais cet instrument est plus suspect que la baguette pou! des tests précis.

D'autre part, en réponse à la deuxième question de M. Foch, M. Rocand confirme que la baguette dont on s'oppose à la chute cassera si elle est rugueuse (coudrier coupée dans un taillis) et glissera ou tournera dans les mains si elle ne l'est pas, à condition toutefois d'avoir un signal prolongé.

M. Rocaro ajoute d'autres détails à sa communication: 1. Dans l'expérience des champs créés artificiellement par un cadre parcouru par un courant électrique, la carte des champs reste semblable a elle-mème si on fait varier le courant, et jamais le signal reçu par l'expérimentateur n'atteint l'impression irrésistible. Si, par contre, on place un deuxième cadre à 3 mètres du premier et si on le fait parcourir par le même courant, l'homme ressent une impression irrésistible qu'on peut relier au fait qu'il est sollicité à deux reprises. Autrement dit, c'est moins la force de l'action physique que sa durée inté- 
grée qui donne cet aspect irresistible que l'on percoit parfois.

$2^{\circ}$ L'effet magnétique, responsable du leurre des sourciers qui a été mentionne dans l'exposé, provient assez souvent des feux de bois : un solide feu de branches dans une forêt fait, sur plusieurs mètres carrés, des cendres lègèrement ferromagnétiques qui influencent les baguettes des sourciers par le mécanisme de la réduction de lhumus.

M. Focr ajoute que la sensibilité dont parlait M. Rocaro est assez répandue : les anciens collaborateurs de M. Garrer. pourraient confimer que, vers 1934, M. GARIEL avait, dans les milieux techniques, la réputation d'être sourcier.

M. Dagallier confirme que M. Garrel avait, en effet, fait beaucoup d'expériences à Lyon avec un sonecier qu'il avait engagé à son service, mais en cherchant à déterminer s'il $y$ avait de l'eau dans un récipient ou non : or, d'après les recherches de $M$. Rocano, l'eau immobile ne donne pas de signal.

M. le Président indique que l'intervention d'un sourcier pourrait être utile pour déceler les fuites d'un barrage : aidé par $M$. Hagcelex, $M$. le Président rappelle qu'un ingénieur, M. Golchaun, avait fait appel aux sourciers, pour un tel cas, a Bouvante, dans le Vercors, il $y$ a trente ou trente-cinc ans, mais sans succès.

M. Dagallier rappelle qu'il a eu une recherche infructueuse dans nne galerie sous l'Obiou.

M. Rocaro indique que, d'après son expérience personnelle, les fuites qu'on a sous les pieds sont celles que l'on détecte le mieux. Dans son live, il a indiqué la situation d'un petit étang bordé par un talus : en se penchant sur le bord de l'étang avec la baguette, on détermine jusqu'où l'eau s'infiltre dans le talus; en piquant des électrodes dans le sol, on trouve, là, de grosses différences de potentiel dues beaucoup à l'argile. Fgalement, en Bretagne, dans des moulins à marée, on a des signaux remarquables en se promenant sur des digues qui subissent des dénivelIations de 5 à $6 \mathrm{~m}$. Toutefois, il lui semble nécessaire d'avoir un milieu bon conducteur autour de la fuite, ce qui ne sera pas le cas pour un barrage.

M. Inmay raconte qu'il $y$ a quinze ans, il a expérimenté avec un géologue qui se servait, non d'une baguetle, mais d'un ressort à boudin. $M$. TRMAY observait ainsi des signaux en se promenant le long d'un pont au-dessus d'un ruisseau, déjà à des distances d'environ $6 \mathrm{~m}$ : le ressort tournait toujours à la même vitesse et commencait sous le même angle. Un électricien éminent, ami de M. ImMay, le professeur OLLENDORFF, suggère une explication de ce phénomène par la radioactivité différentielle de l'eau, par rapport au sol, ce qui pourrait déclencher une réponse physiologique de notre système nerveux qui provoquerait des contractions involontaires de nos muscles produisant cette rotation.

M. Rocaro pense que cela est possible, mais qu'il y a certainement une forme magnétique du déclenchement musculaire.

M. l'ingénieur général Lévy demande si les anomalies dues à des métaux que l'on décèle au magnétomètre sont également décelées par la baguette ou par le pendule.

M. Rocard, qui n'a cité la détection des métaux qu'en l'attribuant aux mineurs allemands en 1450 , croit que c'est I’idée de trouver des filons miniers qui actionnait et pro- voquait alor's l'art des sourciers. Des métaux non ferreux (cuivre ou or) enveloppés dans du plastique ou enterrés ne sont pas détectés par le sourcier. Mais si, enlevant le plastique et la terre étant conductrice, on amorce avec ces métaux une pile de concentration, il n'est nullement exclu qu'on ait des signaux à la condition que le courant produit par cette pile soit assez fort. D'autre part, en bouchant le trou, on remue la terre et on a, peut-être, un humus de caractéristique magnétique différente, susceptible de produire une action magnétique indépendante de la présence physique du cuivre. M. Rocarn croit plutôt que c'est cela.

\section{Rocand poursuit :}

"Les radiesthésistes qui admettent qu'il faut un support physiçue à leur art soutiennent les gens qui découvrent de l'eau, mais ont un doute sur la recherche des métaux, beaucoup trop aléatoire. $\gg$ Telle était du moins l'opinion du Dr Jules Regnault, auteur d'un livre connu.

Répondant à une question sur la détection des mines : II est prodigieux de constater combien de corps, de produits, de matériaux à bon marché sont de petites mines de fer. Par exemple, nos Services de Défense ont étudié des mines non magnétiques enrobées dans un boittier d'ébonite, en les calculant pour qu'elles soient de moins en moins coûteuses. Finalement, ils sont arrivés à quelque chose qui avait des proportions de $1 / 10$ d'oxyde de fer et qui réagissait d'une manière terrible, non pas aux baguettes de sourciers, mais aux détecteurs de mines métalliques. Autrement dit, dans beaucoup de eas, des changements de perméabilité de l'ordre de $1 / 1000^{\circ}$ sont vite atteints. Néanmoins, la mine est finalement trop petite pour que le sourcier y soit sensible, les mines en fer exceptées.

M. Bonnin demande à M. Rocard s'il est certain que les nappes phréatiques qui donnent des signaux ne sont jamais des nappes immobiles ou, tout au moins, que celles-ci coulent assez vite pour produire des potentiels.

M. Rocand répond qu'une grande vitesse d'écoulement n'est pas nécessaire: quand on met le phénomène en équation, le résultat est proportionnel à la pression et la vitesse s'élimine; très souvent, le signal est produit par de l'eau paraissant immobile, mais accompagnée de petites fuites.

M. Rocand insiste sur les forces électromotrices de l'argile. L'an dernier, en Provence, un expérimentateur consciencieux a creusé à $1 \mathrm{~m}-1 \mathrm{~m} 50$ partout où il avait eu des signaux; il a trouvé de l'argile et un peu d'eau dans tous les cas.

M. Breusse, qui fait depuis une quarantaine d'années de la recherche hydrogéologique par géophysique affirme n'avoir que très rarement rencontré des exemples de découverte d'eau en quantité industrielle par radiesthésie, contrairement aux résultats des prospections géophysiques.

M. Rocard est assez de cette opinion. De plus, quand on creuse pour faire un puits, on appelle l'eau qui ne demande qu'à y descendre. On sait, du reste, que les puits ont des interactions entre eux jusqu'a 150 mètres; done, le fait matériel que le puits se remplit d'eau n'est pas forcément à $100 \%$ à l'honneur de celui qui a dit qu'il fallait creuser à cet endroit.

M. le. Président remercie encore vivement M. le professeur ROCARD d'avoir bien voulu nous consacrer une partie de son temps pour nous initier à ce problème, qui a vivement intéressé l'assistance.

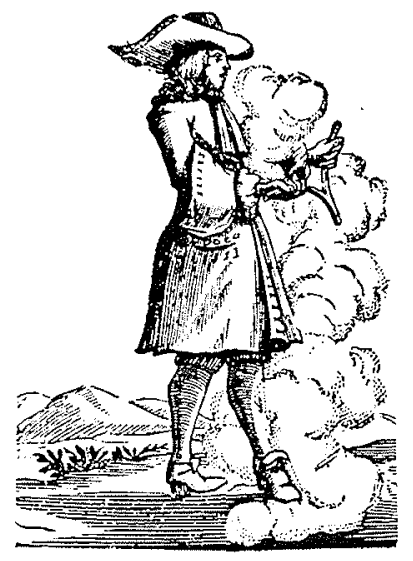






